\title{
Abschied Der letzte der Moguntianer
}

W enn im Herbst mit Hubertus Norbert Riedmiller ,the fastest gun in the West", wie der israelische Professor und Unternehmer Benad Goldwasser den Ultra-Pouchisten Riedmiller nach einer Live-Operation bezeichnete, den Lehrstuhl in seiner „underfränggischen“ Geburtsstadt Herbipolis (Würzburg) räumt und in den urologischen Sonnenuntergang reitet, endet damit nicht nur eine beeindruckende individuelle Karriere. Darüber hinaus findet auch eine Ära herausragender Persönlichkeiten in der deutschen operativen Medizin ihren Abschluss.

Außerdem verliert die internationale Tabakindustrie eine ihrer verlässlichsten Ikonen: Die Frage im Staatsexamen „Stört es Sie, wenn ich rauche?" wurde meines Wissens von keinem der Kandidaten jemals bejaht. Die Spezialdisziplin des mit allen chirurgischen Wassern gewaschenen Urologen ist neben der großen Kinderurologie das sogenannte

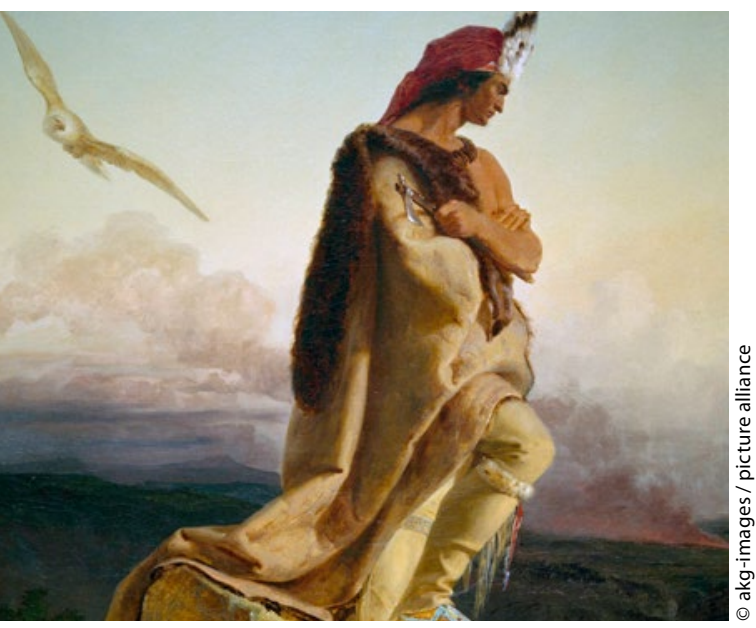

Den letzten Mohikaner erweckte 1826 der Schriftsteller James F. Cooper zum Leben. Mit Hubertus Norbert Riedmiller nimmt nun einer der letzten Anhänger der antirefluxiven Harnleiterimplantation Abschied - sozusagen der letzte Moguntianer.
Troubleshooting, also das unermüdliche und kreative Revidieren andernorts vermurkster Fälle. Bis in die Gegenwart hinein werden diese - begleitet von den guten Wünschen der verzweifelten Ersteingreifer - aus der Nachbarschaft, ganz Deutschland und dem angrenzenden Ausland zu dem Herrgottsschnitzer an den Main geschickt. Gelegentlich sind die Pathologen ratlos, wenn ihnen Großflächenschnitte der Kastanien vorgelegt werden, die er wieder aus dem Feuer geholt hat.

\section{Vom Lummerland ins Main-Florenz} Seit Riedmiller die Appendix vermiformis (als In-Situ-Variante des Mitrofanoff-Prinzips) zu seinem Schicksalsorgan und die Bauhin'sche Klappe zu seiner zweiten Wahl in der Harnableitung gemacht hat, ist er der natürliche Antagonist eines ebenso berühmten orthotopen Ulmer Ferraripiloten mit markantem Haarspoiler. Für viele ist er bis heute auch der Spiritus rector der zuletzt aus der Mode gekommenen antirefluxiven Harnleiterimplantation.

Als einer der jüngsten Ordinarien in der Geschichte der Urologie lehrte der Angehörige der Mainzer Prinzengarde und bekennende James-Bond-Fan zunächst an der ältesten protestantischen Universität der Welt (in dem von Studenten liebevoll Lummerland genannten Marburg), um dann - in der Zeit noch ungewöhnlich und eine Auszeichnung seinen zweiten Lehrstuhl zu erklimmen. Im Jahr 1997 trat der Mann mit dem Stirnband als Markenzeichen die Nachfolge des Cold-Punch-Pioniers und Vornamensvetters Hubert Frohmüller an. Keine leichte Aufgabe übrigens in der Stadt mit dem verunglückten Motto „Provinz auf Weltniveau“: Geht doch der authochtone Würzburger nur zum Sterben an die Uni und bevorzugt ansonsten ein mit der lokalen Presse und dem hohen Klerus herzlich unkritisch verbundenes Tropeninstitut. Oder er strebt an ein kleineres Haus, das weltweit bekannt ist für die assoziierten Weinlagen und die inzwischen leider eingestellte Tradition, seinen Patienten am Abend einen Schoppen zu kredenzen.

\section{Der "Preuße" im wehenden Mantel}

Berüchtigt sind an der Würzburger Klinik nicht nur das preußische Klinikmotto „Arbeyte nur und die Freude kommt von selbst ${ }^{\text {“* }}$ sowie die an das spanische Hofzeremoniell angelehnten Frühbesprechungen (Bericht zur Lage der Station) und der freitägliche Gang durch das Tal der Tränen. Kultstaus genießt bei den Studenten auch „Tante Paula“. Mit dem Prototyp eines Indoor-E-Fahrrads sieht man Riedmiller in zartblauer Operationskleidung und wehendem Visitenmantel noch zu später Stunde die unendlichen Weiten des Zentrums Operative Medizin durchmessen.

Bei der Besetzung von Chefarztpositionen folgte der Katholik („non-strict criteria“) bislang einem subtil diozösanen Muster und beschränkte sich weitestgehend auf Bischofsstädte (Erfurt, Bamberg, Fulda, Stuttgart, Regensburg). Als erster Chairman der Behandlungsleitlinienkommission „Paediatric Urology“ der European Association of Urology zeichnet Riedmiller für die Urversion der oft zitierten Empfehlungen verantwortlich und bot dem aktuellen Machthaber aus Ankara und seinen Unabhängigkeitsbestrebungen lange Paroli.

Jedenfalls fragen sich jetzt alle: Wo schickt man in Zukunft die 72-fach voroperierten Verwachsungsbäuche mit blind endenden Darmschlingen, Fistellabyrinthen und nekrotischem Harnleiter hin?

„Waidmannsdank, Herr Brofesser“!

\section{Prof. Dr. med. Elmar W. Gerharz}

\footnotetext{
* Quelle unbekannt, wird oft Johann Wolfgang von Goethe (stimmt ja auch fast immer) oder Konfuzius zugeschrieben
} 